\title{
LectureCast as 360 Degree Video: What Impact Do Immersion and Presence Experience Have on Learning Performance?
}

\author{
By Andreas Hebbel-Seeger*, Philipp Riehm ${ }^{*}$, \\ André Kopischke ${ }^{\ddagger}$ \& Marianna Baranovskaa ${ }^{\S}$
}

\begin{abstract}
In times of PodCast and MOOC's, a rebirth of the "classical" academic lecture in the form of modern technology-mediated communication as video-conserve can be observed. Current technological developments in the field of audiovisual media reduce the distance between a recorded action and its reception. By using high immersive technologies presence experiences can introduc new qualities of communication. In a study undertaken with freshmen we first explored the everyday use of video technologies by students and their experiences and expectations with regard to their use in formal learning contexts. I addition we investigated the question whether and how learning abilities of the recipients are impacted by immersive technologies (360-degree video on Head Mounted Display/VR headset) compared to classical LectureCast technologies (16:9 Video via Desktop). All things considered, it can be stated that an immersive media format alone does not generate added value: there is no increase of attention that further enhances learning, which can be derived from the feeling (of presence) of sitting in a lecture room, even beyond the possibly initial distraction of the appeal of novelty. Furthermore, the teacher-centered mode is unsuitable for a spherical projection due to its spatial setting, where only one direction is being employed. In order to exploit possible media-specific values of 360-degree video in the context of LectureCasts, it is consequently necessary to work either with a modified concept of a teacher-centered lecture, using the entire space, or to set a focus on the content by combining the recorded lecture with additional visual elements.
\end{abstract}

Keywords: LectureCast, Immersion, Sense of Presence, 360-degree video, VR headset, Video-Learning

\section{Introduction}

Audiovisual media find application in different phases and for different purposes in teaching and learning environments. One of the most common usage scenarios is the illustration and depiction of content and actions; that is, particularly, the recording of lectures in an academic environment. Daily routines of the younger generation can also serve as useful for scientific content (cf. Reinmann 2009, p. 256) by tying in the known media usage behavior of students that, in turn, has been formed by the reception and communication of audio-visual content (Gidion \& Weyrich, 2017, p. 64; Zawacki-Richter, Hohlfeld, \& Müskens, 2014 , p. 32). Thus, on the one hand the use of audio-visual media meets the

\footnotetext{
*Professor, Macromedia University of Applied Sciences, Germany.

${ }^{\dagger}$ Professor, Macromedia University of Applied Sciences, Germany.

\$unior Research Professor, Macromedia University of Applied Sciences, Germany.

${ }^{\S}$ Junior Research Professor, Macromedia University of Applied Sciences, Germany.
} 
demands of contemporary learning opportunities in higher education (cf. Kavanagh et al., 2016, p. 34-35). On the other hand, due to its independence of time and place, it proves adaptability to real-life situations of many students, whose everyday life is characterized by a combination of various requests and activities in the areas of tension between family, professional, and leisure.

Current technological developments in the field of audio-visual media are progressively reducing the distance between a recorded event and its reception. With 360-degree videos, especially in combination with a Head Mounted Display (VR headset), a live situation is increasingly approached by an immersive ${ }^{1}$ projection, which shields the outside world (Slater \& Wilbur, 1997) while aiming for a high presence experience ${ }^{2}$ (Singer \& Witmer, 1998). This in turn gives the recipient the feeling of "truly" being at a different place and possibly also in a different time than is actually physically the case (Ramalho \& Chambel, 2013).

Vohle and Reinmann (2012, p. 3) point out that, while handling a subject of learning in an authentic setting without any pressure to act, it is very likely that one's own knowledge is explained and then also expanded. In this sense, 360degree videos, projected onto a VR headset, receive a special learning potential due to the intended presence experience: learners "dive" into the setting by becoming the center of a spherical (dome-) projection, within which they can analogous to acting in the "real" world- manipulate the image section by moving their heads. At the same time, the physical shield provided by a VR headset (incl. headphones, if applicable) mostly prevents external distractions (Hebbel-Seeger, 2018).

The spherical video recording of a lecture and its projection onto a VR headset represent together a genuine setting, in which the recipient appears to be "truly" present in a lecture auditorium. Concurrently, the content of the lecture becomes abstracted, due to the mere fact of being reproduced on video.

In a nationwide study that occurred with freshmen students in the undergraduate program of Media Management at the Macromedia University of Applied Sciences, we initially explored the usual media usage behavior and student's expectations regarding video usage for learning purposes. In the next step, we examined the question of whether and, if so, to what extent learning abilities of the recipients are impacted by the approach of a real-live-situation in innovative video technology-mediated communication, comparing a "classical" lecture recording on 16:9 video to a 360-degree vision recording.

\footnotetext{
${ }^{1}$ Immersion is a quantifiable technical component whose degree increases with the number of senses addressed. For a depiction to be considered as highly immersive, several criteria must be met: "It includes the extent to which the computer displays are extensive, surrounding, inclusive, vivid and matching" (Slater et al., 1996, p. 3).

${ }^{2}$ Presence in the context of virtual reality refers to the perception of the environment brought about by hardware, whereby mental processes are controlled in an unconscious and conscious manner (Steuer, 1992, p. 75). Within this state, the individual experiences the virtual environment as more engaging than the real world, which is actually surrounding them and thus perceives the content, received as a world being visited rather than images viewed (ibid.).
} 


\section{Video in Teaching and Learning Contexts}

The use of moving images as a supporting measure in the context of learning processes is almost as old as the technology itself. Already at the end of the $19^{\text {th }}$ century, this young medium was used to visualize a learning content, especially so in sports. While the pioneers of chronophotography such as Muybridge and Marey identified the ideal-typical object of the new medium to be cinematic images of animal- and human movements (riders, gymnasts) (cf. Schnelle-Schneyder, 1990, pp. 63-111), at the end of the $19^{\text {th }}$ century, Ernst Kohlrausch applied film footage of high-divers in swimming as visual material (cf. Kemp, 2004). "The vivid interest in chronophotography $[. .$.$] can be partly explained by the eventual success$ to make movements visible that were otherwise not perceivable by the naked eye" ${ }^{3}$ (Verant, 2010). As a result, further technological development in the field of audio-visual media has, above all, effects on the recording and projection qualities, as well as on the availability, accessibility and usability (Hebbel-Seeger, 2018, p. 270): in the course of digitalization, the time periods between a recording and its availability are reduced by several hours, providing a real-time-projection across spatial boundaries. The increased automation of both camera settings and editing software consecutively curtails the requirements for recording and postproduction, thus opening up to even larger user groups. Additionally, camera systems that are becoming increasingly compact and resistant to external influences such as vibrations and moisture, establish new areas of use.

Upon the possibility of displaying space in all directions and at all levels as 360-degree video, substantially new visualization possibilities and didactic options are established. These are prevalent in the immersive character of 360-degree videos, which, with the use of an appropriate end device, keeps the user away from distractive external influences on the one hand and, on the other hand, aims at a presence-experience that, similar to other "lean-forward media" (cf. Katz, 2003, p. 63), leads to an increase of involvement and attention, not least through emotional closeness or connection (Jones, 2007, p. 121; Reyna Zeballos, 2018, p. 1450). If the learning object fills larger areas of a 360-degree production, this format also carries an increased adaptation potential, compared to a "classical" FixFrame ${ }^{4}$ video, because the learners can exploit the (learning) space based on their individual knowledge and interests through a selection of the image.

\section{Study on Media Usage Behavior and Impact}

Not only do we assume that video technologies (can) have a supporting effect in academic teaching and learning environments in various ways, but also that these technologies tie in with the usual media usage behavior of young adults. But how does the academic target group use video technologies for learning purposes in which contexts?

\footnotetext{
${ }^{3}$ Translation by the authors of this paper.

${ }^{4}$ The FixFrame video format is the most commonly used video format with aspect ratios of 16:9 or $4: 3$, which is here considered as a counterpart to the 360-degree or spherical video format.
} 
Therefore, in the underlying study we first examined the video usage behavior of Media Management students at the Macromedia University (Germany). In the second step, we investigated a specific format of mediated teaching and examined whether, and, if so, how the learning performance of recipients is impacted by the approach to a live situation through immersive technologies (360-degree video on head-mounted display/VR headset).

\section{Study Design and Procedure}

The course "Basics of Business Administration" was selected for the study, which is a fundamental part of the program of Media Management, offered at various locations of the Macromedia University of Applied Sciences. The course is compulsory for all students of the Media Management course in the first semester. While the course is usually offered at all locations of the Macromedia University in the form of classroom teaching, students of the winter term 2017/2018 were provided with a lecture recording of the lesson "Materials Management", which was to be received at an individually selected location. A recording of the lecture from the previous year was used, which had been recorded in the presence of students in a conventional seminar room, being made available as a 45-minute video in both formats of 360-degree footage and the "classical" 16:9 format. One of the reasons why the topic "Materials Management" was appropriate for the study is that it was easy to isolate from the rest of the learning content, meaning students were able to participate in the study at a later point of time without losing touch. For the reception of the lecture's content, students were free to choose between a 360-degree presentation via Head Mounted Display (HMD) and a classical 16:9 lecture recording (via monitor), and thus simultaneously assigned themselves to one of the study groups -HMD or Monitor $^{5}$. For the 360-degree video reception, participants of the HMD group received a simple holder ("cardboard"6) for their own mobile device. This combination was to be used as a simple head-mounted display, being held in front of the participants' eyes and applied as a 360-degree video medium in order to receive the lecture recording.

The examination of the students took place in several steps. Initially, the students were asked about their experiences and expectations in terms of videousage for learning purposes and were to assess their subject-specific interest and previous knowledge about the matter compared to their fellow students. In the next step, the students were asked to view the lecture recording using their chosen medium. Subsequently, the knowledge retention of the students was checked via a set of multiple-choice questions about the lecture content and their attitudes towards the experience of the lecture.

\footnotetext{
${ }^{5}$ For better readability, the two groups are referred to from now on as the HMD group (use of 360degree videos via cardboards) and the $M$ group (use of the classical 16:9 format via monitor). ${ }^{6} \mathrm{~A}$ "cardboard" is a holder made of plain cardboard with two lenses into which a smartphone is inserted in order to view video content prepared for 360-degre format.
} 
Both groups received a link to an online questionnaire in which the lecture recordings were integrated (that is, for one group as a "classical" 16:9 FixFrame video and for the other group as a 360-degree content).

\section{Definition of the Study Groups}

Even the first examination of the distribution of both sexes among the test persons in the two study groups clearly shows that the proportion of male test persons in the HMD group ( $\mathrm{n}=91$ ) is very strong with $70.3 \%$. In the $\mathrm{M}$ group $(n=123)$, however, there is a significantly higher proportion of female respondents with $76.4 \%$. This difference can be illustrated by using the chi-square test. It displays a statistically significant correlation between gender and participation mode: $c^{2}(1)=46.527, p<.001, j=.466$. Accordingly, more women selected the reception via the classical video format on a monitor and more men chose the 360degree recording via HMD.

There were also group-specific differences in the choice of reception location (which the students were free to choose). $91.2 \%$ of the subjects from the HMD group $(n=83)$ attended the 360-degree lecture in their private rooms, while the monitor group preferred university as a reception location, with a percentage of $78.9(n=97)$. Due to violations of preconditions, the chi-square test could not be used for statistical testing. The precise Fisher test showed a statistical highly significant difference in the two participation modes regarding the participation environment: $\mathrm{p}<.001$, Cramer-V $=.863$. The effect strength (Cramer-V) is to be classified as high.

On a five-stage Likert scale, the test persons were then able to assess the extent as to which they had been able to follow the lecture recording in an unobstructed participation environment. As the use of head-mounted displays (especially in combination with headphones) largely shields the outside world and thus any disturbing factors, this very item can be used to estimate whether the aforementioned can be considered as a variable of the test result or to be neglected. Here, once again, group-specific differences can be observed. As expected, the subjects of the HMD group $(\mathrm{mw}=1.4)$ concede a higher degree of freedom from interference than the participants of the $M$ group $(m w=1.69)$. This difference becomes significant $(\mathrm{p}=.012)$ with the Mann-Whitney-U-test at a level of $5 \%$. However, with a strength of $\mathrm{r}=.172$, this effect can be described as low. The feeling of freedom from interferences differs in both investigation groups, as the HMD group complies more strongly with the freedom from interference.

Additionally, an attempt was made to identify whether the two study groups vary in terms of their subject-specific interest and previous knowledge in the topic of business studies. This inquiry was supplemented by self-assessments of the respondents with respect to their own performance, interest and commitment in comparison to their fellow students. In the evaluation, no statistically significant differences between the two groups were found - neither concerning subjectspecific interest nor any previous knowledge in the topic of business administration. The self-assessment of respondents compared to their fellow students regarding their own performance, interest and commitment displayed no 
differences between the groups, either: the mean average here lays around the midpoint.

\section{Results on Media Usage Behavior}

As already explained in detail, one of our project purposes is to examine the students' use of video technologies for learning purposes. Therefore, in the first step, we asked the test persons in which situations they had already previously used videos to support their learning interests. The answer indicates practically no differences between the two study groups, which is why we forego a groupspecific evaluation at this point.

Figure 1. Usage of Video as a Learning Aid

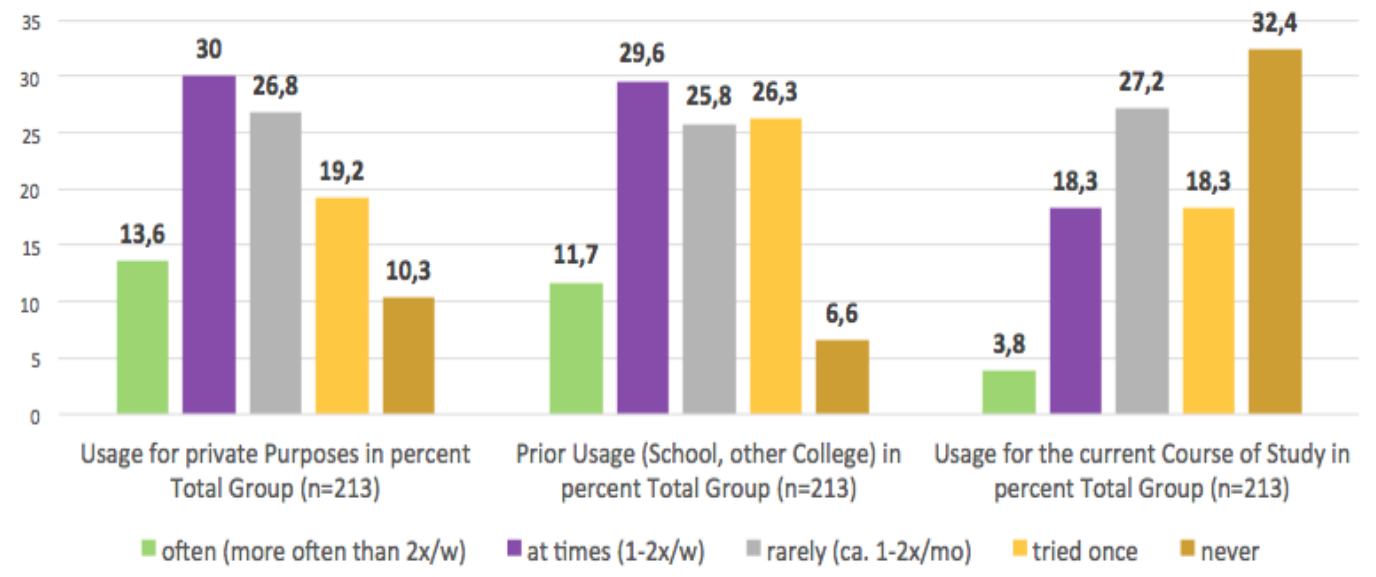

The vast majority of the sample stated that they had already been using videos as a learning aid in formal educational contexts both at school and during professional training, or in a previous course of study, while only one third of the respondents stated that they had gained little or no experience in the discussed formal usage scenarios at all. These findings cannot be applied to the use of video during the imminent course of study. Only less than half of the total sample respondents stated that they had already used learning videos more than once. Compared with the results of the previous question, it can be stated that (so far) learning videos are used less frequently for contemporary formal university educational purposes than have been used for formal educational purposes in the past.

Alongside the use of audiovisual media in formal educational contexts - as previously explained- the use of video for learning purposes in purely informal contexts, meaning students' privateness, should also be depicted. This approach moves closer to the indication of adapting the "usual media usage behavior" of students to more formal educational contexts. Interestingly, there are hardly any differences between the frequency of using videos for learning purposes in prior formal and informal educational contexts. In response to this question, the overwhelming majority of the overall sample respondents affirmed that they had used videos for private learning purposes, as to the frequency of previous use in 
formal contexts just less than $30 \%$ stated that, to date, they had barely used videos or not used them at all.

Figure 2. Hitherto Usage of 360-degree Video via the VR Headset/HMD in \%

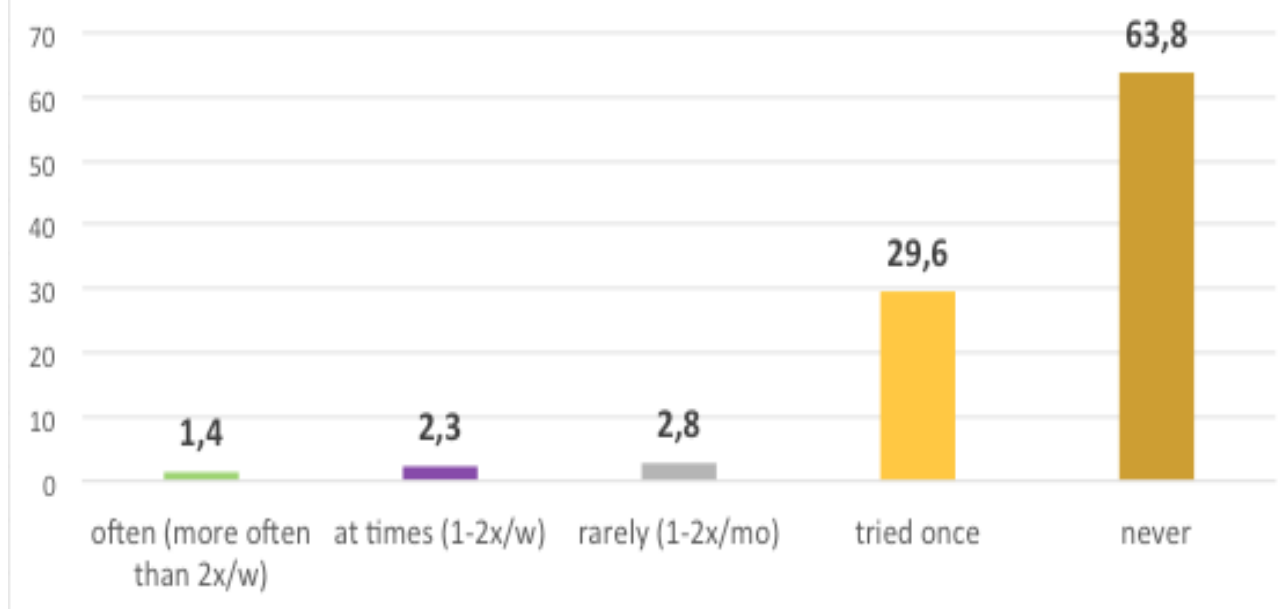

Total Group $(\mathrm{n}=213)$

Particularly, 360-degree videos via the VR headset/HMD have been tested more than once so far by less than $30 \%$ of the test persons in the overall sample (see Figure 2), about $64 \%$ of all test persons denied having done this while only about $7 \%$ have already tried it more than once. In relation to the response behavior of both study groups, no differences can be stated (see Figure 3). In the HMD group $(n=90), 57$ test persons reported that they had never watched 360-degree videos with the VR headset; 28 test persons had tried this at least once.

Figure 3. Data on whether 360-degree Video or the VR Content Already consumed via the VR Headset/HMD Participation Mode in Frequency

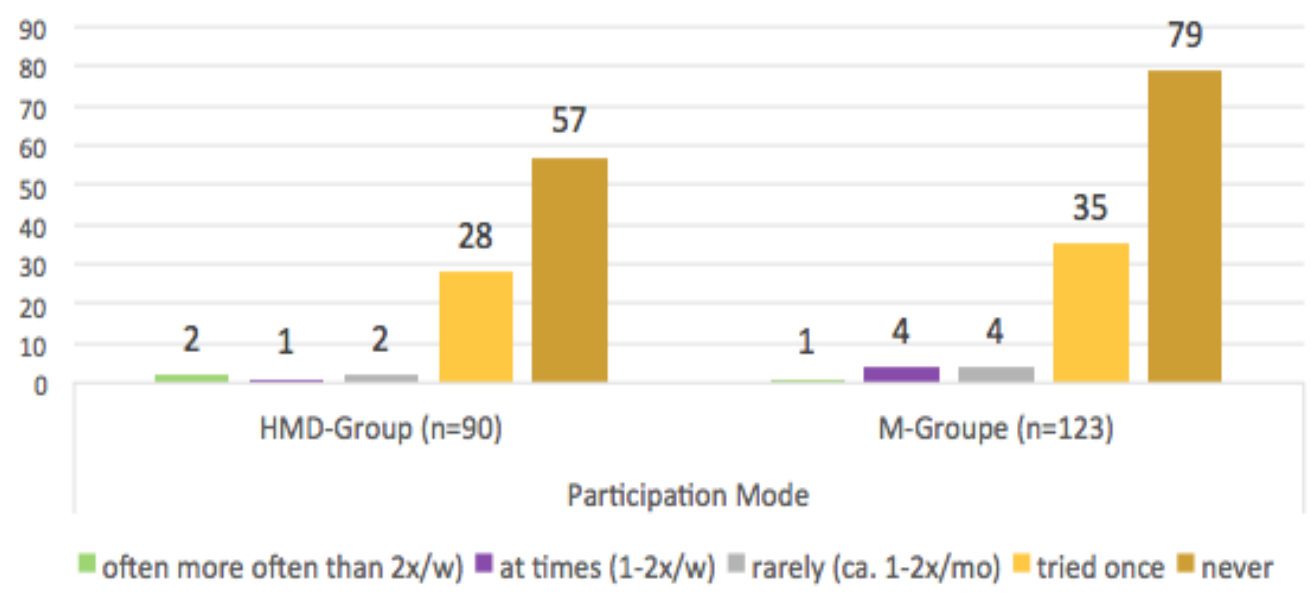

Therefore, for the vast majority of students, the HMD study group's deployment scenario is an innovation. 


\section{Results: The Impact of Immersion and Presence Experience on Learning Performance}

In order to assess the impact that immersion and presence experience have on learning performance, we formed two groups of students as described in a previous section: the Monitor group was offered a lecture recording as a "classical" 16:9 FixFrame video and the HMD group received a 360-degree video treatment. As the participants were free to choose time and place of reception, we were initially interested in whether, and if so, to what extent the lecture recording was consumed. This question was answered by 153 test persons, only 13 of whom stated that they had seen less than half of the video $(n=11$ from the HMD group and $n=2$ from the $M$ group). 61 respondents failed to answer this question ( $n=30$ from the HMD group and $n=31$ from the $M$ group).

Figure 4. Participation Mode of Consume Duration of Educational Videos in Absolute Frequency

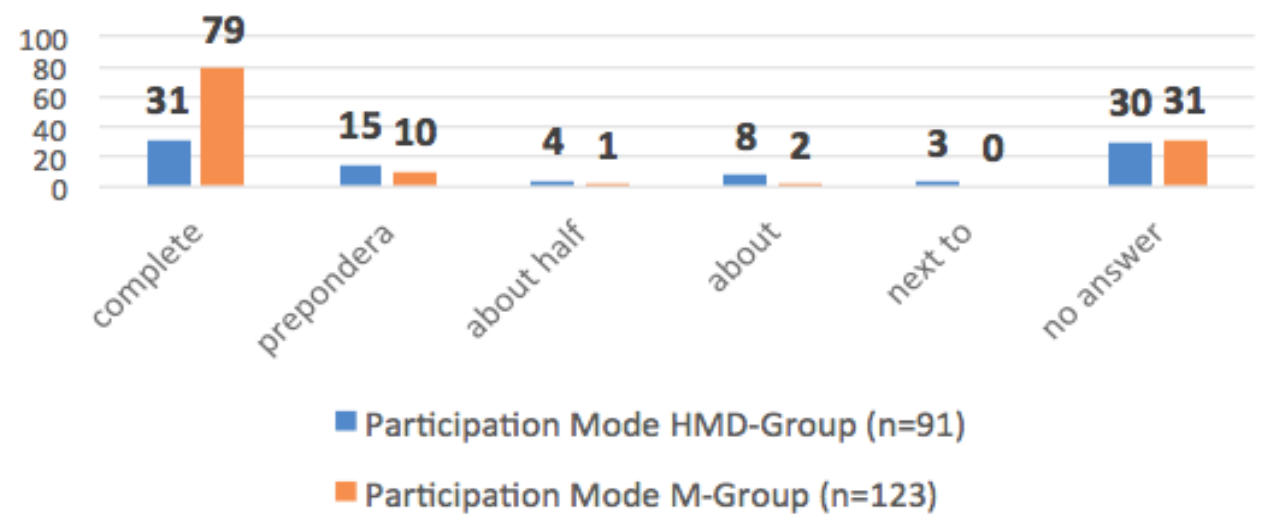

When considering the distributions of the two study groups, it is interesting to note that the proportion of test persons who report having seen the full video is varying between the groups. Compared to the $M$ group, significantly fewer participants of the HMD group watched the video until the end. This is also reflected by the mean ranks: The difference between the mean ranks of HMD (94.06) and $\mathrm{M}$ (65.69) is almost 30. The difference in the duration of consumption between the two study groups can be statistically outlined with the MannWhitney-U test. With $\mathrm{p}<.001$, the group difference is highly significant and, with an effect strength of $\mathrm{r}=.397$, it can be described as medium. Respondents of the $\mathrm{M}$ group watched more of the video than those in the HMD group. 
Figure 5. Absolute Frequency of Break-Off Reasons

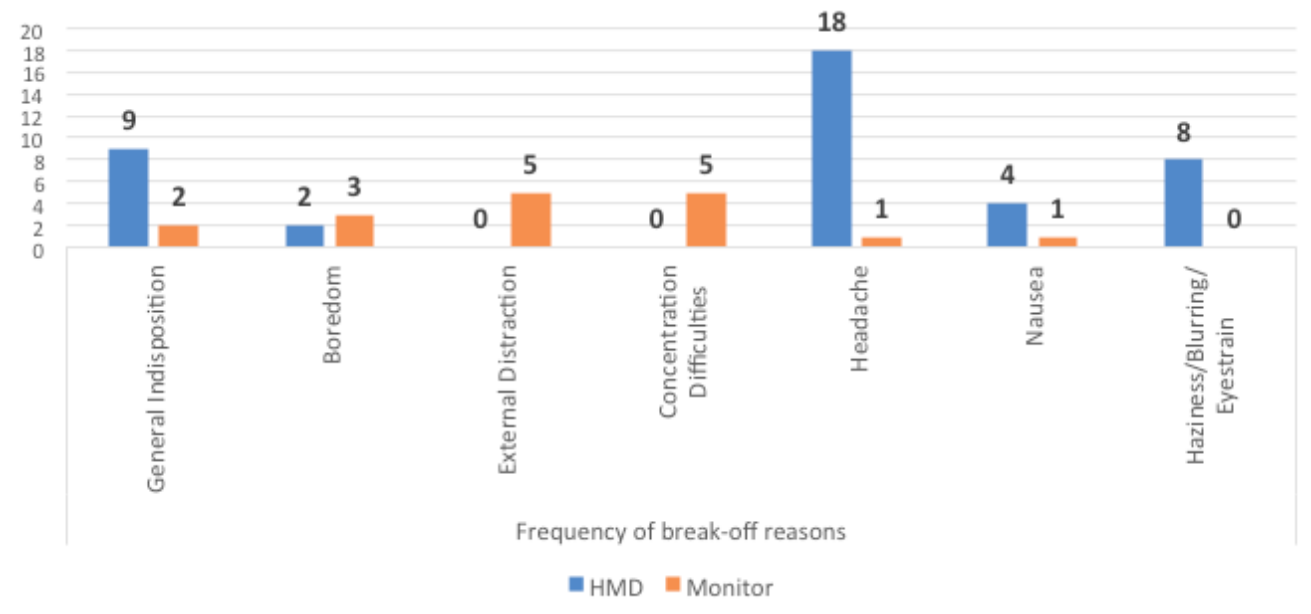

The analysis of the break-off reasons revealed indications for the so-called cybersickness $^{7}$ syndrome in connection with the use of the VR headset. This is clearly displayed by the more frequent statements of HMD group respondents on "general indisposition", "concentration difficulties", "headaches" and "nausea". In addition, the technical quality of the 360-degree video (image resolution) was criticized.

Other mentioned break-off reasons refer to the unhandiness of the cardboard holders and the fact that the simultaneous taking of notes when using the cardboard is just as impossible as the intake of food or beverages.

In order to record the learning performance of the students, the subjects were asked about the content of the videotaped lecture using a multiple-choice questionnaire after the experiment. 58 subjects of the HMD group (corresponding to about 64\%) and 91 subjects of the monitor group (corresponding to $74.8 \%$ ) took part in this survey.

For further evaluation, however, only those test persons who had viewed the respective video "completely" or at least "predominantly" -according to their own explicit statements in the previous assessment - were considered: these comprised of 46 participants in the HMD group and 89 in the M group. Seven questions were to be answered, the order of which corresponds to the chronology of the videotaped lecture. I.e. question 1 related to content discussed at the beginning of the recording, while question 7 referred to content covered at the end of the lecture recording.

From a statistical point of view, questions 2 to 7 were answered equally frequently correct or incorrect by both groups; there was no significant difference in the answers and the assumed learning performance behind them (see Figure 6).

\footnotetext{
${ }^{7}$ Cybersickness, in contrast to motion sickness, only appears during a visual simulation, while motion sickness can appear when viewing sequences that show a movement in conflict with the real physical movement and thus upsets the senses of the receptive system (visual, vestibular, and proprioceptive) (Reason 1978).
} 
Figure 6. Data of Correct Answering in Percentage

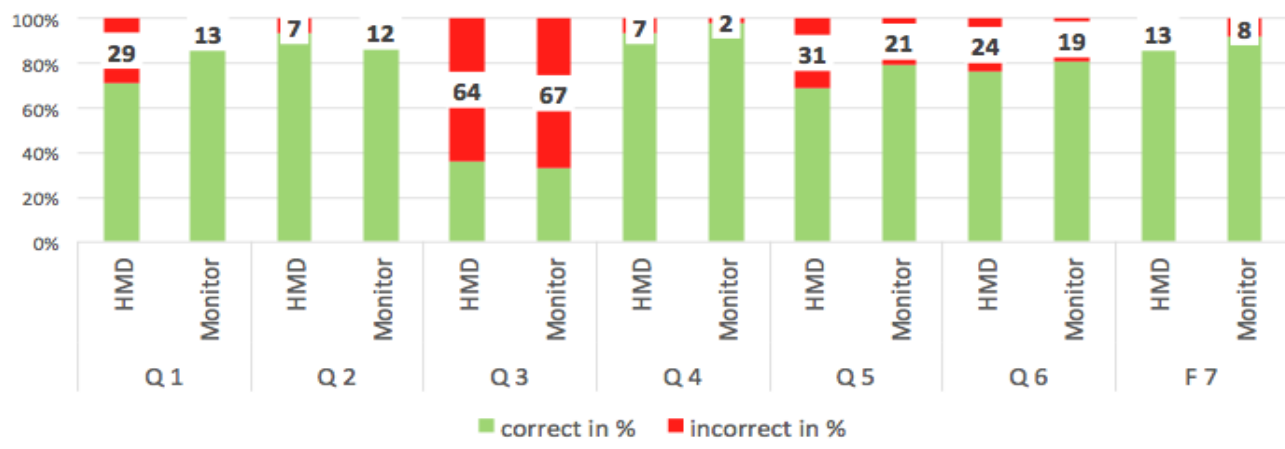

360-degree: $n=45$ and Monitor: $n=89$ (Participation Mode) $^{8}$

Only in question 1 the group belonging correlates with "correct" or "incorrect" answer. The chi-squared test shows a statistically significant correlation between "correct" and "incorrect" answers regarding the participation mode: $\mathrm{c}^{2}(1)=6.590$, $\mathrm{p}=.037$ and an effect strength $\mathrm{j}=.221$ : the $\mathrm{M}$ group successfully solved task 1 significantly more often than the HMD group. Since this difference evens out in progression, we assume an occurrence of a new media-related distraction per attraction at the beginning of the 360-degree reception. As has already been shown, the reception of 360-degree videos using the VR headset is also a novel matter for the vast majority of the HMD group. The aforementioned distraction had already been described by other researchers (Rupp et al., 2016): due to the novelty appeal of 360-degree video and HMD, the test subjects first explore performance and possibilities of the medium instead of concentrating on the media content. Only when this exploration phase is completed, users will focus on the content aspects.

Finally, the participants were questioned about their individual perception and assessment of the learning situation and -performance. In order to compare the learning situations adequately it is not necessary to view the entire 360-degree lecture, but at least about a quarter of the video. For this reason, the following analyses are based on cases where at least a quarter of the session was viewed in a self-chosen participation mode. This applies to 58 HMD group participants and 92 $\mathrm{M}$ group test subjects.

In contrast to the operationalized learning performance, where no groupspecific differences could be demonstrated (with an exception of the question 1, see above), the self-assessment of the test subjects indicates highly significant differences, as can be shown by means of a Mann-Whitney-U test: With $p<.001$ and an effect strength of $r=0.322$, test subjects of the $M$ group assessed their learning performance higher than the participants of the HMD group did.

\footnotetext{
${ }^{8}$ For better comparison we present the data in percent.
} 
Figure 7. Absolute Frequency of Estimation "Compared to Presence Learning Environment in Auditorium I Felt Less Distracted"

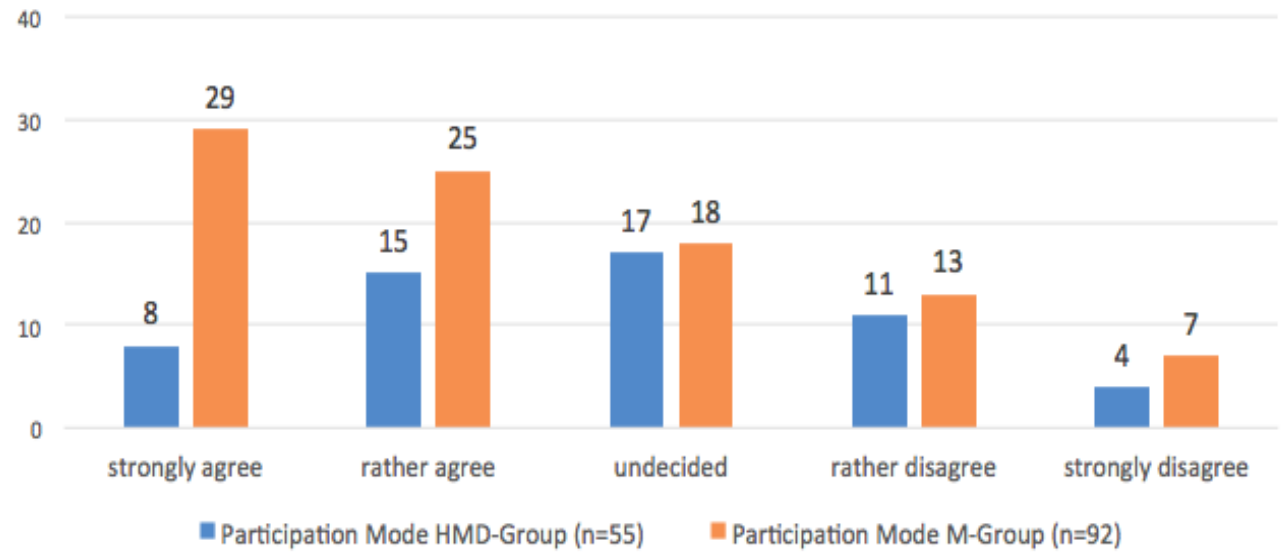

This result correlates with the answers to the question "I consider the media format being used today (16:9 video or 360-degree video) as well suited for the reception of a lecture recording". In the HMD study group, the mean value of 3.04 was significantly higher ("undecided") than in the M group with 2.24 ("rather agree"). The $M$ group therefore found the reception of the 16:9 format more suitable than the HMD group examined the reception of "their" 360-degree recordings. In addition, test subjects of the $M$ group stated that they were less distracted than participants of the HMD group, compared to the situation in the auditorium.

Even though the statistical examination using the Mann-Whitney-U test displays a minor effect $(\mathrm{p}=.041, \mathrm{r}=.169)$, the result is intriguing. Before the reception of the instructional video, participants of the HMD group felt less disturbed with in their participation environment compared to the monitor group (see description of the study groups). Either the participants of the HMD group were disturbed during the video reception (which mainly took place at home), or the 360-degree setting created a feeling of distraction (see above), which, however, stands in contrast to the immersive character of the very media format. At the same time, $M$ group participants found it easier to stay alert and focused in comparison to HMD-group respondents.

The question as to whether participants would rather decide in favor of today's used media format for learning purposes in the future or not also indicates a higher acceptance in the $\mathrm{M}$ group ( $\mathrm{mw}=2.26$; "rather agree") than in the HMD group (mw=3.42; "undecided"). It must be pointed out that 114 of a total 123 participants in the $\mathrm{M}$ group had little or no experience with 360-degree video and were unable to collect such during the treatment. Therefore, their answers merely reflect their expectations regarding the use of 360-degree video with HMD without being able to make a real comparison. 


\section{Conclusion}

Students use videos for learning purposes in different contexts. However, our survey conveys that this is not (yet) the case in academic learning processes. Additionally, we found that the reception of 360-degree video via HMD's was a new experience for the vast majority of students. Since videos carry supportive potential for learning processes, it is important to systematically explore and expand the possibilities of the usage.

In this sense, by representing the underlying study of this paper, we were interested whether and to what extent the immersive video format of a 360-degree projection on a HMD differs from the "classical" lecture cast in 16:9 formats on a monitor, in relation to the resulting learning success as well as the self-perception of the learning situation. As a result, we were not able to detect any significant differences between the two partial samples. In general, it remains to be questioned whether the chosen form of self-assessment per multiple-choice survey is a suitable procedure for measuring learning success.

Additionally, we assume that the simple design and thus limited usability of the cardboards have had an impact on the survey results. The resolution of 360degree videos (especially considering the playback quality of the smartphones used by the students) is also a factor that cannot be neglected if, for example, texts or graphics are shown in the lecture.

Overall, in our consideration, the depiction of a teacher-centered lecture in a 360-degree video is not a suitable didactic scenario. It emerges that an immersive media format alone does not generate any added value. The recording of a lecture as a 360-degree video and its projection on the HMD represents an authentic setting, in which the recipient is given the feeling of "truly" sitting in a lecture room. At the same time, however, there is an elemental distance to the "actual" learning object as it is "only" conveyed orally and, where applicable, by the use of accompanying media (usually slide presentations). Regarding the learning situation, a lecture recording as a 360-degree video is therefore highly "authentic", however, this is not the case when considering this particular learning object.

A teacher-centered lecture is unsuitable for a spherical projection due to its spatial setting, as the space is not fully used and merely "exploited" in one direction. In order to release the media-specific added value of 360-degree video in the context of LectureCasts, it is necessary to a) either create a teaching concept that includes and expands the full space usage for the reception, to recycle it as annotation surfaces for instance (Vohle \& Reinmann, 2012), b) or to focus on the "authenticity" of the learning object by visualizing of the 360-degree video in relation to lecture cast in the form of a "hypervideo" for example (Chambel et al. 2011, p. 1). 


\section{References}

Chambel, T., Chhaganlal, M. N., Neng, L. A. R. (2011). Towards immersive interactive video through $360^{\circ}$ hypervideo. In Proceedings of the $8^{\text {th }}$ International Conference on Advances in Computer Entertainment Technology, 78 (ACE '11). ACM, New York, NY, USA.

Gidion, G., Weyrich M. (Eds.) (2017). Mediale Hochschul-Perspektiven 2020 in BadenWürttemberg: empirische Untersuchung im Rahmen der Allianz "Forward IT" [Media University Perspectives 2020 in Baden-Württemberg: Empirical Study within the Alliance "Forward IT"]. KIT Scientific Publishing: Karlsruhe. Retrieved from https://bit.ly/2P8XkRm. [Accessed 12 Mars 2019].

Hebbel-Seeger, A. (2018). 360-Video in Trainings- und Lernprozessen. In U. Dittler \& C. Kreidl (eds.), Hochschule der Zukunft-Beiträge zur zukunftsorientierten Gestaltung von Hochschulen, (pp. 265-290). Berlin, Heidelberg: Springer VS.

Jones, M. T. (2007). Presence as external versus internal experience: How form, user, style, and content factors produce presence from the inside. In Proceedings of the Tenth Annual International Meeting of the Presence Workshop. Barcelona: ISPR. 115-126. Retrieved from https://bit.ly/2Psxo20. [Accessed 25 January 2019].

Katz, H. E. (2003). The media handbook: A Complete guide to advertising media selection, planning, research, and buying (2. Aufl.). Mahwah, NJ: Lawrence Erlbaum Associates.

Kavanagh, S., Luxton-Reilly, A., Wüensche, B., Plimmer, B. (2016). Creating $360^{\circ}$ educational video: a case study. In Proceedings of the $28^{\text {th }}$ Australian Conference on Computer-Human Interaction (OzCHI '16). (pp. 34-39) New York, NY, USA: ACM. doi: $10.1145 / 3010915.3011001$.

Kemp, C. (2004). The chronophotographer Ernst Kohlrausch and the physics of gymnastics. In Bernard Finn (ed.) Artefacts: Studies in the history of science and technology, Bd. 4 - Presenting Pictures, (pp. 43-69). London: NMSI Trading Ltd.

Ramalho, J., \& Chambel, T. (2013). Immersive $360^{\circ}$ mobile video with an emotional perspective. Proceedings of ImmersiveMe 2013 (pp. 35-40). Bacelona/Spain: ACM.

Reinmann, G. (2009). iTunes statt Hörsaal? Gedanken zur mündlichen Weitergabe von wissenschaftlichem Wissen [iTunes instead of lecture hall? Thoughts on the oral transmission of scientific knowledge]. In N. Apostolopoulos, H. Hoffmann, V. Mansmann \& A. Schwill (Eds.), E-Learning 2009- Lernen im digitalen Zeitalter (pp. 256-267). Münster: Waxmann.

Reyna Zeballos, J. (2018). The potential of 360-degree videos for teaching, learning and research. In INTED2018 Proceedings. Valencia: IATED Academy. 1448-1454.

Rupp, M.A., Kozachuk, J., Michaelis, J R., Odette, K.L., Smither, J.A., \& McConnell, D.S. (2016). The effects of immersiveness and future VR expectations on subjectiveexperiences during an educational $360^{\circ}$ video. Proceedings of the Human Factors and Ergonomics Society Annual Meeting, 60(1), 2108-2112. https://doi.org/10.1177/ 1541931213601477. [Accessed 16 February 2019].

Schnelle-Schneyder, M. (1990). Photographie und Wahrnehmung am Beispiel der Bewegungsdarstellung im 19 [Photography and perception using the example of the representation of movement in the $19^{\text {th }}$ century]. Jahrhundert, Marburg: Jonas.

Singer, M.J., \& Witmer, B.G. (1998). Measuring presence in virtual environments: a presence questionnaire. presence: Teleoperators and Virtual Environments, 7(3), 225240.

Slater, M., Linakis, V., Usoh, M., Kooper, R., Street, G. (1996). Immersion, presence, and performance in virtual environments: an experiment with tri-dimensional chess. In 
ACM Virtual Reality Software and Technology (VRST), (pp. 163-172). New York, NY: ACM Press.

Slater, M., \& Wilbur, S. (1997). A framework for immersive virtual environments (FIVE): Speculations on the role of presence in virtual environments. Presence: Teleoperators and Virtual Environments 6, 6, 603-616.

Steuer, J. (1992). Defining virtual reality: dimensions determining telepresence. Journal of Communication, 42(4), 73-93. http://dx.doi.org/10.1111/j.1460-2466.1992.tb00812.x.

Verant, O. (2010). Die Bewegungsdarstellung in der Fotografie des 19. Jahrhunderts- von der wissenschaftlichen Analyse zum künstlerischen Ausdruck [The representation of movement in 19th century photography - from scientific analysis to artistic expression]. Fotogeschichte, 30(116). Retrieved from https://bit.ly/2RFvmOX. [Accessed 12 Mars 2019].

Vohle, F., \& Reinmann, G. (2012). Förderung professioneller Unterrichtskompetenz mit digitalen Medien: Lehren lernen durch Videoannotation [Promotion of professional teaching skills with digital media: learning lessons through video annotation]. In R. Schulz-Zander, B. Eickelmann, H. Moser, H. Niesyto \& P. Grell, (eds.), Jahrbuch Medienpädagogik 9, (pp. 413-429). Berlin, Heidelberg: Springer VS.

Zawacki-Richter, O., Hohlfeld, G., \& Müskens, W. (2014). Mediennutzung im Studium [Media usage in studies]. Schriftenreihe Zum Bildungs- Und Wissenschaftsmanagement, 1(1), 1-36. Oldenburg: Carl von Ossietzky Universität Oldenburg. Retrieved from https://bit.ly/2PDoiQk. [Accessed 12 Mars 2019] 